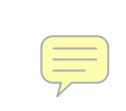

\title{
Quantum symmetries in the free field realization of $\mathrm{W}_{n}$ algebras
}

\author{
R. Cuerno ${ }^{1,2}$ \\ Instituto de Fisica Fundamental, CSIC, Serrano 123, E-28006 Madrid, Spain
}

\begin{abstract}
$\mathrm{W}_{n}$ algebras are considered in their free field representation to show that they are endowed with a quantum group symmetry which is a $\mathbb{Z}_{2}$ twist à la Drinfel'd of $\mathscr{U}_{q_{+}}(\operatorname{sl}(n)) \times \mathscr{U}_{q_{-}}(\mathrm{sl}(n))$. We use the contour picture of quantum groups due to Gómez and Sierra. A sample computation for the $\Re$ matrix is also performed.
\end{abstract}

\section{Introduction}

There are two subjects in the realm of current theoretical physics whose interest is growing very rapidly. One of them is the mathematical structure of quantum groups [1], recently found in exactly solvable lattice models (see ref. [2] and references therein), factorizable $S$-matrix models [3], quantum Liouville theory [4] and 2D conformal field theory (CFT) [5]. Concerning this last issue Gómez and Sierra have been able to uncover the quantum group symmetry behind the $c<1$ rational CFTs, by the use of contour deformation techniques [6]. The same procedure has been also successfully applied to WZNW models $[7,8]$ and $N=1,2$ superconformal field theories [9].

The second subject is that of $\mathrm{W}_{n}$ algebras. First found in 2D CFT $[10,11]$, they also appear in the contexts of integrable theories in $1+1$ dimensions (KdV type models, Toda theories), IRF models or random multi-matrix models (for a brief review with references see ref. [12]).

In this letter we use the Gómez-Sierra (GS) contour techniques [6] in the context of the free field realization of $\mathrm{W}_{n}$ algebras $[10,11]$ to show that here the underlying quantum group symmetry is related via a twist à la Drinfel'd [13] to a quantum algebra $\mathscr{T}_{q_{+}}(\operatorname{sl}(n)) \otimes \mathscr{U}_{q_{-}}(\operatorname{sl}(n))$. We also compute as an example the $\mathscr{R}$ matrix in the fundamental representation of the $\mathrm{W}_{3}$ algebra and find agreement with the standard $\mathscr{R}$ matrix in the fundamental representation for $\mathscr{U}_{q_{+}}(\mathrm{sl}(3))$. This $\mathscr{R}$ matrix computation should not be confused with the braiding matrices for chiral vertex operators which are worked out in ref. [14] also by means of contour manipulation techniques (with a different choice of contours, though).

\section{Free field realization of $\mathrm{W}_{n}$ algebras}

In this section we fix the notation and normalization to be used in the rest of the letter.

The free field (or Feigin-Fuchs) construction of the $\mathrm{W}_{n}$ algebras $[10,11]$ uses $n-1$ bosonic fields $\phi(z)=\left(\phi_{1}(z), \ldots, \phi_{n-1}(z)\right)$ [remember sl $(n)$ has $n-1$ simple roots ]

$$
\left\langle\phi_{i}(z) \phi_{j}(w)\right\rangle=-2 \delta_{i j} \ln (z-w)
$$

1 Supported by a FPI grant by the Spanish MEC.

2 E-mail address: IMTRC59@CC.CSIC.ES. 
(we shall always restrict to the holomorphic sector) and a background charge at infinity. We shall take all the simple roots $\left\{\boldsymbol{e}_{i}\right\}_{1}^{n-1}$ of $\mathrm{sl}(n)$ to have squared length equal to 2 . The Cartan matrix elements then read

$\mathscr{K}_{a b}=\boldsymbol{e}_{a} \cdot \boldsymbol{e}_{b}=2 \delta_{a, b}-\delta_{a, b+1}-\delta_{a, b-1}$.

We will also introduce the $n-1$ fundamental weights $\left\{\boldsymbol{\omega}_{a}\right\}_{1}^{n-1}$ such that

$e_{a} \cdot \omega_{b}=\delta_{a b}, \quad \mathscr{G}_{a b}=\omega_{a} \cdot \omega_{b}$

with $\mathscr{G}$ the inverse of the Cartan matrix $\mathscr{K}$. The stress tensor reads

$W^{(2)}(z)=T(z)=-\frac{1}{4} \partial \phi(z) \cdot \partial \phi(z)+\mathrm{i} \alpha_{0} \rho \cdot \partial^{2} \phi(z)$.

$\rho$ is taken to be equal to half the sum of the positive roots of $\operatorname{sl}(n)$ and so $\rho=\sum_{a=1}^{n-1} \omega_{a}$. One can construct $W^{(k)}(z)$ fields of spins $k=3,4, \ldots, n$ and show that their modes indeed close what is called a $\mathrm{W}_{n}$ algebra, that includes Virasoro as a subalbegra $[10,11]$. One can also construct vertex operators

$V_{\beta}(z) \equiv: \exp [\mathrm{i} \beta \cdot \phi(z)]:$

with conformal weight $\Delta_{\boldsymbol{\beta}}=\boldsymbol{\beta} \cdot\left(\boldsymbol{\beta}-2 \alpha_{0} \boldsymbol{\rho}\right) . \boldsymbol{\beta}$ is a vector whose components are $n-1$ constant coefficients. With these vertices we can construct screening operators in the spirit of the Feigin-Fuchs construction of DotsenkoFateev [15]. Seeking the conformal weight to have value +1 we find two families of solutions

$J_{a}^{ \pm}(z)=: \exp \left[\mathrm{i} \alpha_{ \pm} e_{a} \cdot \phi(z)\right]:, \quad a=1, \ldots, n-1$

with

$\alpha_{+}+\alpha_{-}=\alpha_{0}, \quad \alpha_{+} \alpha_{-}=-\frac{1}{2}$.

Their having weight +1 makes the screening charges

$Q_{a}^{ \pm}=\oint_{\mathrm{C}} \mathrm{d} z J_{a}^{ \pm}(z)$

well defined objects whose commutators with any of the generators of the $\mathrm{W}_{n}$ algebra give boundary terms $[10,11]$. If the contours $\mathrm{C}$ do not cross branch cuts, the boundary terms vanish so that the $Q_{a}^{ \pm}$commute with all the generators of the $\mathrm{W}_{n}$ symmetry. If there exist branch cuts we will integrate along the GS contours [6] and keep track of the appearing boundary terms.

The screening operators enable us to construct null vectors $[10,11]$. The completely degenerate representations are those in which there exist enough of them to completely determine the correlation functions. They are those whose highest weight $\boldsymbol{V}_{\boldsymbol{\beta}}$ has $\boldsymbol{\beta}$ given by $[10,11]$

$\boldsymbol{\beta}=\sum_{a=1}^{n-1} \omega_{a}\left[\left(1-n_{a}\right) \alpha_{+}+\left(1-m_{a}\right) \alpha_{-}\right]$.

These are the ones we shall be dealing with in the sequel. We end up this section by recalling the basic braiding relations among vertex operators, which will be needed below. They are $[10,11]$

$V_{\beta}(z) V_{\gamma}(w)=\exp (2 \pi \mathrm{i} \boldsymbol{\beta} \cdot \gamma) V_{\gamma}(w) V_{\beta}(z)$. 


\section{The representation space}

We are going to build a representation space for the quantum group symmetry out of the elements seen in the previous section, namely vertex operators and screening charges.

Take a vertex $V_{\beta}(z)$ with $\beta$ satisfying (9) for some set of integers $\left\{n_{a}, m_{a} \geqslant 1\right\}_{a=1}^{n-1}$. We associate to it the representation space ${ }^{\beta}$ consisting of the following vectors:

$e_{p}^{\beta_{p}^{+}, I_{s}^{-}}(z) \equiv \int_{\mathrm{C}_{p}} \mathrm{~d} t_{p} J_{i_{p}^{+}}^{+}\left(t_{p}\right) \ldots \int_{\mathrm{C}_{1}} \mathrm{~d} t_{1} J_{i_{1}^{+}}^{+}\left(t_{1}\right) \int_{\mathrm{C}_{s}^{\prime}} \mathrm{d} t_{s}^{\prime} J_{i_{s}^{-}}^{-}\left(t_{s}^{\prime}\right) \ldots \int_{\mathrm{C}_{1}^{\prime}} \mathrm{d} t_{1}^{\prime} J_{i_{1}^{-}}^{-}\left(t_{1}^{\prime}\right) V_{\beta}(z)$,

where $I_{p}^{+} \equiv\left\{i_{p}^{+}, \ldots, i_{1}^{+}\right\}, I_{s}^{-} \equiv\left\{i_{s}^{-}, \ldots, i_{1}^{-}\right\}$, and $\left\{i_{j}^{+}\right\} P_{1}^{p},\left\{i_{k}^{-}\right\}_{1}^{s}$ can take any of the values 1 to $n-1$. We will call $r_{a}^{ \pm}$the number of times the screening $J_{a}^{ \pm}$appears in (11). The contours of integration are those of GS [6] and the order in which we have written the $J_{a}^{+}$'s with respect to the $J_{a}^{-}$'s differs from other possibilities [due to (10) ] by signs which will be unimportant to us. One can check for different examples that the dimension of this space is finite; for instance, if we take for $\mathrm{W}_{3}$

$\beta=-\alpha_{+} \omega_{1}$

one can see that this space consists of only three vectors,

$e_{0}(z) \equiv V_{\beta}(z), \quad e_{1}(z) \equiv \int \mathrm{d} t J_{1}^{+}(t) V_{\beta}(z), \quad e_{21}(z) \equiv \int \mathrm{d} t \mathrm{~d} u J_{2}^{+}(t) J_{1}^{+}(u) V_{\beta}(z)$,

by passing to the path-ordered representation of GS. Since we are going to identify the quantum group generators with contour creation and destruction operators acting on $\boldsymbol{y}^{\beta}$, the complete proof of the soundness of our definition of representation space will rely on the fulfilment of the $q$-deformed Serre relations for such generators (see refs. [7,16] and references therein). Then let us first define

$F_{a}^{+} e_{I_{D}^{+}, I_{s}^{-}}^{\beta}(z) \equiv e_{I_{D+1}^{+}, I_{s}^{-}}^{\beta}(z), \quad F_{a}^{-} e_{I_{D}^{+}, I_{s}^{-}}^{\beta}(z) \equiv e_{I_{p}^{+}, I_{s+1}}^{\beta}(z)$.

Note that $F_{a}^{ \pm}$increases the number of contours along which the screening $J_{a}^{ \pm}(z)$ is integrated by one (contour creation operators). On the other hand we apply to the vector (11) a Virasoro operator $L_{n}$ to get

$$
\begin{aligned}
& L_{n}\left(e_{I_{p}^{+}, I_{s}}^{\beta}(z)\right)=e_{I_{p}^{+}, I_{s}}^{\beta}\left(L_{n} V_{\beta}(z)\right)-\sum_{j=1}^{p} \lim _{t \rightarrow \infty} t^{n+1} J_{i_{j}^{+}}^{+}(t) B_{i_{j}^{+}}^{+} e_{I_{p}^{+}-1, I_{s}}^{\beta}(z) \\
& \quad-\sum_{k=1}^{s} \lim _{t \rightarrow \infty} t^{n+1} J_{i \bar{k}}^{-}(t) B_{i_{\bar{K}}}^{-} e_{I_{p}^{+}, I_{s-1}}^{\beta}(z),
\end{aligned}
$$

where

$I_{p-1}^{+}=\left\{i_{p}^{+}, \ldots, i_{j+1}^{+}, i_{j-1}^{+}, \ldots, i_{1}^{+}\right\}, I_{s-1}^{-} \equiv\left\{i_{s}^{-}, \ldots, i_{\bar{k}+1}, i_{\bar{k}-1}, \ldots, i_{1}^{-}\right\}$,

$B_{i_{j}^{+}}^{+} \equiv q_{+}^{\sum_{m=j+1}^{p} *_{i m}^{+} i_{j}^{+}}\left[1-\exp \left(4 \pi \mathrm{i} \alpha_{+} \boldsymbol{\beta} \cdot \boldsymbol{e}_{i_{j}^{+}}\right) q_{+}^{\left.2 \sum_{m=1}^{j-1} \cdot x_{i_{j}+i_{m}^{+}}\right]}\right.$

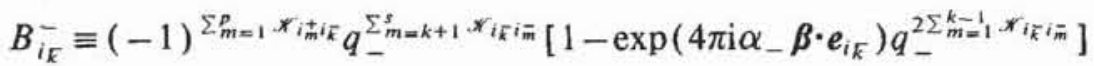

with $q_{ \pm} \equiv \exp \left(2 \pi \mathrm{i} \alpha_{ \pm}^{2}\right)$. Now, if we define

$L_{n}\left(e_{I_{p}^{+}, I_{5}^{-}}^{\beta}(z)\right) \equiv e_{l_{p}^{+}, I_{\bar{s}}}^{\beta}\left(L_{n} V_{\beta}(z)\right)-\sum_{a=1}^{n-1}\left[A_{+} \lim _{t \rightarrow \infty} t^{n+1} J_{a}^{+}(t) E_{a}^{+}+A_{-} \lim _{t \rightarrow \infty} t^{n+1} J_{a}^{-}(t) E_{a}^{-}\right] e_{I_{b}^{+}, I_{5}^{-}}^{\beta}(z)$,

we are left with the definition of the action of the contour destroying operators on our representation space: 


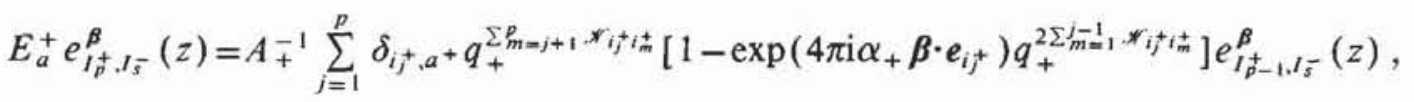

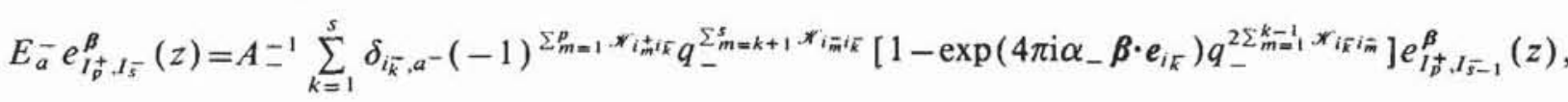

with $I_{p-1}^{+}, I_{s-1}^{-}$as in (16), $A_{ \pm}$are simply normalization constants. Now we can prove the Serre relations. The procedure is exactly as in ref. [8], so that here we will just quote the result. It is

$\sum_{k=0}^{1-\mathscr{X}_{a b}}(-1)^{k}\left\{\begin{array}{c}1-\mathscr{K}_{a b} \\ k\end{array}\right\}_{q^{ \pm}}\left(F_{a}^{ \pm}\right)^{1-\varkappa_{i j-k}} F_{b}^{ \pm}\left(F_{a}^{ \pm}\right)^{k}=0$

$(a \neq b)$, and an analogous one for the $\left\{E_{a}^{ \pm}\right\}$[just replace in (19) $F^{\prime}$ 's by $E$ 's]. We use the definitions

$\{a\}_{q} \equiv \frac{q^{a}-q^{-a}}{q-q^{-1}}, \quad\left\{\begin{array}{l}a \\ b\end{array}\right\}_{q}=\frac{\{a\}_{q} !}{\{b\}_{q} !\{a-b\}_{q} !}$

with $\{a\}_{q} !=\{a\}_{q}\{a-1\}_{q} \ldots\{1\}_{q}$.

\section{The quantum group}

We just need one further definition concerning the operators in the Cartan subalgebra. Given a screened vertex operator such as (11) redefine coefficients so that $\beta=-2 \sum_{a=1}^{n-1} \omega_{a}\left(j_{a}^{+} \alpha_{+}+j_{a}^{-} \alpha_{-}\right)$. Then introduce the following operators:

$\boldsymbol{H}^{ \pm} e_{I_{p}^{+}, I_{s}^{-}}^{\beta}(z) \equiv 2 \sum_{a=1}^{n-1}\left(j_{a}^{ \pm} \omega_{a}-\frac{1}{2} r_{a}^{ \pm} e_{a}\right) e_{I_{p}^{ \pm}, I_{s}^{-}}^{\beta}(z)$,

so that

$H_{a}^{ \pm} \equiv \boldsymbol{e}_{a} \cdot \boldsymbol{H}^{ \pm}$

Now it is an easy task to compute the commutation relations of the quantum group operators $\left\{F_{a}^{ \pm}, E_{a}^{ \pm}, K_{a}^{ \pm} \equiv\right.$ $\left.q_{ \pm}^{-H_{d}^{\frac{1}{d}}}\right\}$ on our representation space, with the following result:

$F_{a}^{+} F_{\bar{b}}=(-1)^{x_{a b}} F_{\bar{b}} F_{a}^{+}, \quad E_{a}^{+} E_{\bar{b}}^{-}=(-1)^{x_{a b}} E_{b}^{-} E_{a}^{+}, \quad E_{a}^{ \pm} F_{b}^{\mp}=(-1)^{x_{a b}} F_{b}^{\mp} E_{a}^{ \pm}$,

$E_{a}^{ \pm} F_{b}^{ \pm}-q_{ \pm}^{* a b} F_{b}^{ \pm} E_{a}^{ \pm}=\delta_{a b} A_{ \pm}^{-1}\left[1-\left(K_{a}^{ \pm}\right)^{2}\right], \quad\left[K_{a}^{ \pm}, K_{b}^{ \pm}\right]=\left[K_{a}^{ \pm}, K_{b}^{\mp}\right]=0$,

$K_{a}^{ \pm} F_{b}^{ \pm}=q_{ \pm}^{\text {*ab }} F_{b}^{ \pm} K_{a}^{ \pm}, \quad K_{a}^{ \pm} E_{b}^{ \pm}=q_{ \pm}^{-x_{a b}} E_{b}^{ \pm} K_{a}^{ \pm}, \quad\left[K_{a}^{ \pm}, F_{b}^{\mp}\right]=\left[K_{a}^{ \pm}, E_{b}^{\mp}\right]=0$.

The comultiplication rules can also be computed by contour deformation arguments [6]. One gets

$\Delta\left(K_{a}^{ \pm}\right)=K_{a}^{ \pm} \otimes K_{a}^{ \pm}, \quad \Delta\left(F_{a}^{ \pm}\right)=F_{a}^{ \pm} \otimes 1+K_{a}^{ \pm} \exp \left(\pi \mathrm{i} H_{a}^{\mp}\right) \otimes F_{a}^{ \pm}, \quad \Delta\left(E_{a}^{ \pm}\right)=E_{a}^{ \pm} \otimes \mathfrak{v}+K_{a}^{ \pm} \exp \left(\pi \mathrm{i} H_{a}^{\mp}\right) \otimes E_{a}^{ \pm}$.

Two comments are in order. First there appear some signs in the relations (23) that prevent us from having a properly direct product (as far as the commutation relations are concerned) between the + and the - sets of operators. Second, the comultiplication rules (24) also contain some unusual factors that mix the + and operators. The first point can be solved through the following redefinitions:

$\varphi_{a}^{ \pm} \equiv \exp \left( \pm \frac{1}{2} \pi \mathrm{i} H_{a}^{\mp}\right) F_{a}^{ \pm}, \quad \epsilon_{a}^{ \pm} \equiv \exp \left(\mp \frac{1}{2} \pi \mathrm{i} H_{a}^{\mp}\right) E_{a}^{ \pm}$.

Then one gets the following algebra of operators:

$\left[\varphi_{a}^{ \pm}, \varphi_{b}^{\mp}\right]=\left[\epsilon_{a}^{ \pm}, \epsilon_{b}^{\mp}\right]=\left[\varphi_{a}^{ \pm}, \epsilon_{b}^{\mp}\right]=0, \quad\left[K_{a}^{ \pm}, \varphi_{b}^{\mp}\right]=\left[K_{a}^{ \pm}, \epsilon_{b}^{\mp}\right]=\left[K_{a}^{ \pm}, K_{b}^{\mp}\right]=0, \quad\left[K_{a}^{ \pm}, K_{b}^{ \pm}\right]=0$, 
$K_{a}^{ \pm} \varphi_{b}^{ \pm}=q_{ \pm}^{\text {*ab }} \varphi_{b}^{ \pm} K_{a}^{ \pm}, \quad K_{a}^{ \pm} \epsilon_{b}^{ \pm}=q_{ \pm}^{- \text {Xab }} \epsilon_{b}^{ \pm} K_{a}^{ \pm}, \quad \epsilon_{a}^{ \pm} \varphi_{b}^{ \pm}-q_{ \pm}^{\text {Xab }} \varphi_{b}^{ \pm} \epsilon_{a}^{ \pm}=\delta_{a b} A_{ \pm}^{-1}\left[-\left(K_{a}^{ \pm}\right)^{2}\right]$.

( 26 cont'd)

The $q_{ \pm}$-deformed Serre relations (19) still hold for the operators $\varphi_{a}^{ \pm}, \epsilon_{a}^{ \pm}$. The algebra of operators $\mathrm{Q}$ defined by (26) is a direct product of two copies (labeled + and - ) of a same algebra $\mathscr{U}_{q}(\operatorname{sl}(n))$. As for the comultiplication rules one gets

$\Delta\left(K_{a}^{ \pm}\right)=K_{a}^{ \pm} \otimes K_{a}^{ \pm}, \quad \Delta\left(\varphi_{a}^{ \pm}\right)=\varphi_{a}^{ \pm} \otimes \exp \left( \pm \frac{1}{2} \pi \mathrm{i} H_{a}^{\mp}\right)+K_{a}^{ \pm} \exp \left[\pi \mathrm{i}\left(1 \pm \frac{1}{2}\right) H_{a}^{\mp}\right] \otimes \varphi_{a}^{ \pm}$,

$\Delta\left(\epsilon_{a}^{ \pm}\right)=\epsilon_{a}^{ \pm} \otimes \exp \left(\mp \frac{1}{2} \pi \mathrm{i} H_{a}^{\mp}\right)+K_{a}^{ \pm} \exp \left[\pi \mathrm{i}\left(1 \mp \frac{1}{2}\right) H_{a}^{\mp}\right] \otimes \epsilon_{a}^{ \pm}$.

It can be checked that these $\triangle$ 's provide an algebra homomorphism of (26); moreover, if we define the counit $\epsilon$ and the antipodal map $\gamma$ as

$\epsilon\left(K_{a}^{ \pm}\right)=1, \epsilon\left(\epsilon_{a}^{ \pm}\right)=\epsilon\left(\varphi_{a}^{ \pm}\right)=0, \epsilon(\mathbb{1})=1$,

$\gamma\left(K_{a}^{ \pm}\right)=K_{a}^{ \pm}, \quad \gamma\left(\varphi_{a}^{ \pm}\right)=-\left(K_{a}^{ \pm}\right)^{-1} \varphi_{a}^{ \pm}, \quad \gamma\left(\epsilon_{a}^{ \pm}\right)=-\left(K_{a}^{ \pm}\right)^{-1} \epsilon_{a}^{ \pm}$,

then all the Hopf algebra axioms are satisfied for Q, i.e., one has

$(\Delta \otimes \mathrm{id}) \Delta(a)=(\mathrm{id} \otimes \Delta) \Delta(a), \quad m(\operatorname{id} \otimes \gamma) \Delta(a)=m(\gamma \otimes \mathrm{id}) \Delta(a)=\epsilon(a) 1$,

$(\epsilon \otimes \mathrm{id}) \Delta(a)=(\mathrm{id} \otimes \epsilon) \Delta(a)=a$

for $a$ any of the $\left\{\varphi_{a}^{ \pm}, \epsilon_{a}^{ \pm}, K_{a}^{ \pm}\right\} . m$ is the multiplication map in Q. Finally, we still notice that the comultiplication rules of $Q$ contain factors which mix the + and the - pieces of the algebra. We can define a new comultiplication $\widetilde{\Delta}$ by means of an element $F \in \mathrm{Q} \otimes \mathrm{Q}$ given by [13]

$F=\exp \left(\frac{\pi \mathrm{i}}{2} \sum_{a, b=1}^{n-1} \mathscr{G}_{a b}\left(H_{a}^{+} \otimes H_{b}^{-}-H_{a}^{-} \otimes H_{b}^{+}\right)\right)$,

$\widetilde{\Delta}(a)=F \Delta(a) F^{-1}$

such that the new algebra $\widetilde{Q}$ is $\mathscr{U}_{q_{+}}(\operatorname{sl}(n)) \otimes \mathscr{U}_{q_{-}}(\operatorname{sl}(n))$. We can say that $\mathrm{Q}$ is a twist of $\widetilde{Q}$ [13]. The $\tilde{\phi}$ operator acting on $\tilde{Q} \otimes \tilde{Q} \otimes \widetilde{Q}$ and which enters the definition of the twist [13] can be shown to be equal to the identity, so that $\widetilde{Q}$ is actually a Hopf algebra, and not quasi-Hopf as would have been the case if $\tilde{\phi} \neq \mathrm{id}$ [13].

\section{The $\mathscr{R}$ matrix}

We close this letter with a sample computation for the $\mathscr{R}$ matrix in the representation space (13); for the $\boldsymbol{\beta}$ in (12) (remember this was for a $\mathrm{W}_{3}$ algebra) and with a basis ordering for our $\left.\mathscr{Y}^{\boldsymbol{\beta}}\left\{e_{0} z\right), e_{21}(z), e_{1}(z)\right\}$ it can be shown using the techniques devised in ref. [6], that

$\tilde{e}_{m_{1}}\left(z_{1}\right) \otimes \tilde{e}_{m_{2}}\left(z_{2}\right)=\exp \left(-\frac{2}{3} \pi \mathrm{i} \alpha_{+}^{2}\right) \sum_{m_{1}^{\prime}, m_{2}^{\prime}} \tilde{e}_{m_{2}^{\prime}}\left(z_{2}\right) \otimes \tilde{e}_{m_{1}^{\prime}}\left(z_{1}\right) \mathscr{R}_{m_{1}^{\prime} m_{2}^{\prime} ; m_{1} m_{2}}$

with $\mathscr{R}$ the usual $\mathscr{R}$ matrix for $\operatorname{sl}(3)_{q_{+}}$in the fundamental representation (the vectors $\left\{\tilde{e}_{i}\right\}$ are just the pathordered version of the $\left\{e_{i}\right\}$ in (13) [6]) 


$$
\mathscr{R}=\left(\begin{array}{ccccccccc}
q_{+} & 0 & 0 & 0 & 0 & 0 & 0 & 0 & 0 \\
0 & 0 & 0 & 1 & 0 & 0 & 0 & 0 & 0 \\
0 & 0 & 0 & 0 & 0 & 0 & 1 & 0 & 0 \\
0 & 1 & 0 & q_{+}-q_{+}^{-1} & 0 & 0 & 0 & 0 & 0 \\
0 & 0 & 0 & 0 & q_{+} & 0 & 0 & 0 & 0 \\
0 & 0 & 0 & 0 & 0 & 0 & 0 & 1 & 0 \\
0 & 0 & 1 & 0 & 0 & 0 & q_{+}-q_{+}^{-1} & 0 & 0 \\
0 & 0 & 0 & 0 & 0 & 1 & 0 & q_{+}-q_{+}^{-1} & 0 \\
0 & 0 & 0 & 0 & 0 & 0 & 0 & 0 & q_{+}
\end{array}\right) .
$$

The fact that the $\mathscr{R}$ matrix for $\mathrm{W}_{n}$ algebras has been shown in a different context [17] not to be the standard one (though the same eigenvalues still occur) could be related to the existence of a different twist of the Hopf algebra $\widetilde{Q}$ in such realizations of the relevant conformal field theory.

\section{Acknowledgement}

It is a pleasure to thank Professor G. Sierra for suggesting the problem and for many patient discussions and explanations. I would also like to thank F. Jiménez and M. Ruiz-Altaba for discussions, and the Theory Division at CERN for hospitality while this work was being completed.

\section{References}

[1] V.G. Drinfel'd, Quantum groups, in: Proc. Intern. Congress of Mathematicians (Berkeley, 1986), Vol. I (American Mathematical Society, Providence, RI, 1985) p. 798;

M. Jimbo, Lett. Math. Phys. 10 (1985) 63; 11 (1986) 247.

[2] H. Saleur and J.B. Zuber, Integrable lattice models and quantum groups, lectures given at the 1990 Trieste Spring School on String theory and quantum gravity, preprint SPhT/90-071 (1990).

[3] A. Le Clair, Phys. Lett. B 230 (1989) 103;

F.A. Smirnov and N. Reshetikhin, Hidden quantum group symmetry and integrable perturbations of conformal field theories, Harvard preprint HUTMP 89 B246 (1989);

T. Hollowood, A quantum group approach to constructing factorizable $S$-matrices, University of Oxford preprint OUTP-90-15P (1990).

[4] T. Hollowood and P. Mansfield, Nucl. Phys. B 330 (1990) 720;

J.L. Gervais, Commun. Math. Phys. 130 (1990) 257; 138 (1991) 301; Phys. Lett. B 243 (1990) 85.

C. Gómez and G. Sierra, Phys. Lett. B 255 (1991) 51.

[5] L. Alvarez-Gaumé, C. Gómez and G. Sierra, Nucl. Phys. B 319 (1989) 155; B 330 (1990) 347; Phys. Lett. B 220 (1989) 142;

G. Moore and N.Yu. Reshetikhin, Nucl. Phys. B 328 (1989) 557;

E. Witten, Nucl. Phys. B 330 (1990) 285;

V. Pasquier and H. Saleur, Nucl. Phys. B 330 (1990) 523.

[6] C. Gómez and G. Sierra, Phys. Lett. B 240 (1990) 149; Nucl. Phys. B 352 (1991) 791.

[7] C. Ramírez, H. Ruegg and M. Ruiz-Altaba, Phys. Lett. B 247 (1990) 499; The quantum symmetry of conformal field theories, in: Proc. XIV Johns Hopkins Workshop (Debrecen, 1990), eds. G. Domokos et al. (World Scientific, Singapore, 1991).

[8] C. Ramirez, H. Ruegg and M. Ruiz-Altaba, Nucl. Phys. B, to appear.

[9] F. Jiménez, Phys. Lett. B 252 (1990) 577; EUITA-DA preprint (June 1991).

[10] A.B. Zamolodchikov, Theor. Mat. Phys. 65 (1985) 1205; V.A. Fateev and A.B. Zamolodchikov, Nucl. Phys. B 280 (FS 18) (1987) 644.

[11] V.A. Fateev and S.L. Lykyanov, Intern. J. Mod. Phys. A 3 (1988) 507.

[12] A. Bilal, Introduction to W-algebras, lectures given at the 1991 Trieste Spring School on String theory and quantum Gravity, CERN and LPTENS preprint CERN-TH.6083/91, LPTENS 91/10 (April 1991). 
[13] V.G. Drinfel'd, Quasi Hopf algebras and Knizhnik-Zamolodchikov equations, in: Problems of modern quantum field theory, eds. A.A. Belavin, A.U. Klimyk and A.B. Zamolodchikov, Research Reports in Physics (Springer, Berlin, 1989) p. 1. [14] A. Bilal, Nucl. Phys. B 330 (1990) 399; Intern. J. Mod. Phys. A 5 (1990) 1881.

[15] V.S. Dotsenko and V.A. Fateev, Nucl. Phys. B 240 (1984) 312; B 251 (1985) 691.

[16] P. Bouwknegt, J. McCarthy and K. Pilch, Phys. Lett. B 234 (1990) 297; Commun. Math. Phys. 131 (1990) 125.

[17] E. Cremmer and J.L. Gervais, Commun. Math. Phys. 134 (1990) 619. 